\title{
CARACTERÍSTICAS DE PACIENTES DISFÁGICOS EM SERVIÇO DE ATENDIMENTO DOMICILIAR PÚBLICO
}

\author{
Carina Teixeira PAIX Ã Oa ${ }^{a}$ Lolita D opico da SILVA ${ }^{b}$
}

\section{RESUM 0}

Este artigo teve como objetivo identificar as características dos pacientes portadores de disfagia decorrente de um acidente cerebrovascular em relação à idade, fatores de risco, episódios de acidente vascular cerebral (AVC) e grau de dificuldade para deglutir em função da consistência dos alimentos. Estudo transversal com 30 pacientes através da técnica da entrevista. Os resultados mostraram média de idade de $73,6( \pm 9,55)$ anos, sendo $76,47 \%$ de mulheres, todos hipertensos e a metade diabéticos (58,82\%). Todos tiveram acidente vascular isquêmico. N ão se encontrou associação entre disfagia e idade nem com a frequência de episódios de AVC. Pacientes com hipertensão e diabetes apresentam maior chance para a disfagia. Constatou-se que a dificuldade de deglutição para líquidos é maior que os pacientes com disfagia são idosos com vários fatores de risco diante do que o enfer meiro deve orientar para uma al imentação segura diminuindo complicações como a pneumonia e a desnutrição.

D escritores: D eglutição. A cidente cerebral vascular. Assistência domiciliar.

\section{RESUMEN}

E ste artículo tuvo como objetivo identificar las características de pacientes portadores de disfagia en virtud de un accidente cer ebrovascular, en relación a la edad, factores de riesgo, episodios de accidente cer ebrovascular y nivel de dificultad para la deglutición de acuerdo con la consistencia de alimentos. E studio transversal con muestra de treinta pacientes y técnica de entrevista. L os resultados muestran media de edad de 73,6 ( $\pm 9,55$ ) años con $76,47 \%$ de mujeres, todos eran hipertensos y la mitad diabéticos (58,82\%). Todos tuvier on accidente cer ebrovascular isquémico. N o se encontró asociación entre disfagia y edad, tampoco con la cantidad de episodios de accidente cerebrovascular. Pacientes hipertensos y diabéticos tienen mayor probabilidad para la disfagia. P resentan más dificultad para deglutir líquidos. Todos son de edad con varios factores de riesgo fundamentando la idea de que es importante que el enfer mero garanticeuna al imentación segura evitando la neumonía y desnutrición. Conclusión, la disfagia necesita ser precoz mente evaluada en virtud de sus complicaciones.

Descriptores: D eglución. Accidente cer ebrovascular. A tención domiciliaria de salud.

T ítulo: Características de pacientes disfági cos en un servicio de atención domiciliar público.

\section{ABST RACT}

This article discusses the characteristics of dysphagic patients victims of a stroke. The objectives were to identify the profile of patients with dysphagic and measure its association with age, risk factors, episodes of stroke and consistency of food. As a methodology, we used the cross- sectional study sample of thirty patients. R esults show that all 17 dysphagic patients were hypertensive and more than half also diabetic (58.82\%). All of them had ischemic stroke. Therewas no association betw een dysphagic and age, neither with the frequency of stroke episodes. Patients with hypertension and diabetes had higher odd ratio for dysphagic refer ring to the swallowing of liquids. All of them were elderly with three risk factors (hypertension, diabetes and obesity) and with increased difficulty in swallowing liquids. As a conclusion, it was observed that the nurse needs to be trained to recognize dysphagia and its complications, such as pneumonia and malnutrition.

Descriptors: D eglutition. Stroke $\mathrm{H}$ ome nursing.

Title: Characteristics of dysphagic patients in public home care service.

\footnotetext{
a M estre em Enfer magem, Enfer meira do Instituto N acional de Cardiologia (INCL) e do H ospital Pró-Cardíaco, Rio de Janeiro, Brasil.

${ }^{b}$ D outora em Enfermagem, Professora Adjunta do Programa de Pós-G raduação em Enfermagem da U niversidade Estadual do Rio de Janeiro (UERJ), Coordenadora do Curso de Especialização em Enfermagem Intensivista da UERJ, Rio de Janeiro, Brasil.
} 


\section{INT RODUÇÃO}

Acidente Vascular Cerebral (AVC), também chamado de doença silenciosa do século, apresenta alta mortalidade e morbidade sequelar importante por longo prazo. A parece como a primeira causa de invalidez e morte e com uma consequência importante que são as incapacidades dos pacientes vítimas desta doença(1).

Outro aspecto relevante repousa na importância epidemiológica do AVC, tendo em vista que é a terceira causa de mortalidade em países industrializados e a primeira causa de incapacidades em adultos. N o Brasil o AVC é a primeira causa de internações, mortalidade e incapacidades, superando até mesmo 0 câncer e as doenças cardíacas ${ }^{(2)}$.

$M$ ais da metade dos pacientes após um AVC tem entre seis e dez tipos de incapacidades, sendo a mais comum a fraqueza muscular presente em $77,4 \%$ deles, seguida dos distúrbios da comunicação e linguagem e da disfagia. E sta última responde por $44,7 \%$ das incapacidades encontradas ${ }^{(2)}$.

Incapacidade é uma restrição, resultante de uma deficiência, da fal ta de habilidade para desempenhar uma atividade considerada normal para 0 ser humano. A disfagia aparece como uma incapacidade que contribui para a perda da funcionalidade e independência para al imentar-se, trazendo riscos de desnutrição e pneumonia aspirativa para 0 paciente $\mathrm{e}^{(3)}$.

A disfagia consiste na dificuldade da deglutição por perda na progressão ordenada do al imento da boca para o estômago ${ }^{(1)}$. T rata-se do sintoma de uma doença em curso, podendo ocorrer em qualquer idade de forma imprecisa. E $m$ função da localização do déficit estrutural, pode ser classificada em disfagia orofaríngea que vai da boca até 0 esfíncter cricofaríngeo e disfagia esofágica aquela abaixo do esfíncter cricofaríngeo.

A disfagia or ofaríngea pode ser resultado de intercorrências locais, neurológicos ou musculares, exemplo, quando uma disfunção do esfíncter esofágico superior ocorre prejudicando a habilidade de transferir a comida da boca ao esôfago. A disfagia esofagiana é o resultado de qualquer obstrução mecânica (como tumor) do órgão ou alteração na mobilidade, que afeta a própria função muscular do esôfago. Já a disfagia obstrutiva mostrase como uma dificuldade na deglutição de sólidos, progredindo para o líquido, no curso da doença. A disfagia que resulta de disfunção neuromuscular, como no caso do AVC é apresentada com problemas na deglutição de líquidos e sólidos ${ }^{(1)}$.

Cerca de 30 a $40 \%$ dos sobreviventes no primeiro ano após o AVC requerem al gum tipo de auxílio para a alimentação decorrente da disfagia. A perda de autonomia para alimentação entre adultos e a sua consequente dependência é uma forma de expressão da gravidade desta incapacidade resultante do AVC(3).

A frequência e gravidade da disfagia após o AVC e a necessidade do pronto reconhecimento e tratamento adequado constituem um desafio, tanto pel o impacto à saúde, quanto pelas repercussões na vida das pessoas e suas famílias. A disfagia pode acarretar prejuízos nos aspectos nutricionais, de hidratação, no estado pulmonar, prazer alimentar e social do indivíduo ${ }^{(1)}$.

A incoordenação do mecanismo de deglutição pode resultar em aspiração de al imentos ou regurgitação nasal. A ssim a disfagia, geralmente é associada com tosse após a deglutição, dificuldade respiratória, infecção pulmonar, comprometendo tanto a parte respiratória do indivíduo como a sua nutrição. As principais metas do enfermeiro com o paciente e a família incluem 0 al cance de uma dieta 0 mais próxima do habitual do paciente antes do AVC, mantendo-o com ausência de infecções pulmonares por broncoaspiração e livre de desnutrição.

A adaptação à disfagia após um AVC é um processo de colaboração entre o paciente, família e profissionais de saúde e o cuidado domiciliar ressurge então como uma das possibilidades de promover mudanças na qualidade de atenção à saúde ${ }^{(1)}$.

No Brasil, o Serviço de A tendimento D omiciliar (SAD) criou uma nova modalidade de assistência com o objetivo de atender, no domicílio, pessoas portadoras de doenças crônico-degenerativas e/ ou com incapacidade funcional provisória ou permanente ${ }^{(4)}$. N esse cenário, encontra-se o paciente com disfagia decorrente de um AVC.

Conhecer as características dos pacientes com disfagia decorrente de um AVC e atendidos em um serviço domiciliar público, pode contribuir para o direcionamento de um cuidado seguro pelo enfermeiro levando à diminuição das reinternações. Sabe-se que um entre três pacientes sofrerá entre uma ou duas reinter nações no primeiro ano após um AVC e a maior causa de reinternação são as infecções pulmonares decorrentes da disfagia(4).

No âmbito da enfermagem, são necessárias pesquisas que permitam conhecer as respostas 
humanas dos pacientes a quem os enfermeiros prestam cuidados para programar ações de enfermagem seguras e de qualidade. Os estudos dessa natureza contribuirão com todos aquel es que procuram traçar os resultados do trabal ho da enfermagem com os seus indicadores de segur ança e qualidade da assistência e com medidas que funcionem como barreiras para evitar a pneumonia e a desnutrição, aumentando a segurança da deglutição em pacientes disfágicos.

F undamentada por essas questões, foi desenvolvido uma pesquisa que teve como objetivo identificar as características dos pacientes portadores de disfagia em decorrência de um AVC em relação à idade, fatores de risco, episódio de AVC e dificuldade de deglutição em função da consistência dos alimentos.

\section{MET ODOLOGIA}

Tratou-se de um estudo do tipo transversal feito no SAD do H ospital G eral de Jacarepaguá (HJ) no município do Rio de Janeiro. Os estudos transversais caracterizam-se por colher informações em um único momento de uma população em função de uma característica em comum e são apropriados para descrever características de determinadas variáveis e seus padrões de distribuição. A população do SAD deste hospital era de 112 pacientes todos adultos vítimas de AVC.

0 cálculo amostral foi feito a partir da fórmula utilizada para cálculo amostral de estudos transver sais de população finita (até 100.000), usando-se um nível de confiança de $95 \%$, um a de 0,05 e um valor crítico de 1,96, dado pela fórmula ( $2 \alpha /$ $2=1,96)$, considerando-se que $20 \%$ dos pacientes podiam ter disfagia. 0 valor de $20 \%$ foi estimado a partir de um banco de dados preexistente na instituição onde os dados foram coletados, equival endo a trinta e cinco pacientes entre os quais houve cinco perdas finalizando então trinta pacientes.

U sou-se a técnica de amostragem sistemática selecionando um prontuário a cada quatro da listagem de pacientes vitimas de AVC. A técnica de coleta de dados foi a entrevista estruturada e quando o paciente apresentava sequela que comprometia a cognição ou a comunicação, a entrevista foi realizada junto ao responsável do paciente.

As entrevistas foram realizadas de acordo com a agenda do programa SAD, onde previamente são marcadas as visitas entre as equipes e os familiares responsáveis pelos pacientes em atendimento. 0 acesso à agenda do SA D ocorreu mediante a autorização da instituição responsável pelo programa.

0 instrumento de coleta de dados foi dividido em duas partes: dados do perfil sócio-laborialsaúde e dados da disfagia. A tendendo a Resolução 196/ 96 do Consel ho N acional de Saúde sobre pesquisa em seres humanos ${ }^{(5)}$, todos assinaram o Termo de Compromisso Livre e Esclarecido. A pesquisa foi aprovada pelo Comitê de Ética do M unicípio do Rio de Janeiro com o no 43/ 07. A coleta foi realizada no período de agosto a setembro de 2008. Os dados receberam tratamento estatístico com o uso do programa Statistical Package for the Social Sciences (SPSS), versão 14, e com a ajuda de um profissional estatístico da U niversidade do E stado do Rio de Janeiro.

\section{RESULTADOS}

\section{Características dos pacientes disfágicos}

Do total de trinta pacientes vítimas de AVC, foi confirmada a disfagia em dezessete destes definindo uma prevalência de $57 \%$. A média de idade foi de 73,6 $( \pm 9,55)$ com uma mediana de 75 anos e moda de 78 anos. 0 predomínio de pacientes com disfagia encontrou-se na faixa etária entre 60-79 anos (36,67\%). A idade mínima foi de 45 anos (01 sujeito) e a máxima de 88 anos (02 sujeitos).

A maioria dos pacientes eram mulheres (76,47\%) com predomínio do ensino fundamental completo (76,48\%). Quanto à situação laboral, observou-se entre os disfágicos que doze $(70,59 \%)$ eram aposentados. Todos os pacientes eram hipertensos e mais da metade $(58,82 \%)$ também diabéticos. Todos os pacientes com disfagia tiveram AVC isquêmico e dez (58,82\%) pacientes tiveram um único episódio de AVC e sete $(41,18 \%)$ pacientes tiveram dois episódios.

$\mathrm{N}$ a amostra de trinta pacientes vítimas de AVC ao comparar os pacientes com disfagia $(n=17)$ e sem disfagia ( $n=13)$, obser vou-se que entre os pacientes sem disfagia, também houve predomínio de mulheres (23,33\%) com a idade entre 60 a 79 anos. No que se refere à escolaridade, também se confirmou o predomínio do ensino fundamental entre pacientes não disfágicos. Quanto à situação de trabalho, entre os pacientes sem disfagia um encon- 
trava-se empregado e o restante estava aposentado. $O$ AVC do tipo hemorrágico apareceu em um único paciente sem disfagia. Quanto ao número de episódios de AVC, tanto para o grupo com e sem disfagia foi semelhante, pois a maioria teve um único episódio de AVC.

E m relação ao tipo de AVC, todos os pacientes disfágicos tiveram AVC do tipo isquêmico apesar do grau de dificuldade de deglutição poder estar relacionado com o tipo de AVC. T anto o AVC isquêmico quanto o hemorrágico podem causar disfagia $a^{(1,6,7)}$.
Os pacientes vítimas de AVC foram separados segundo os fatores de risco encontrados (hipertensão, diabetes e obesidade) e segundo a presença ou não de disfagia. Os fatores de risco preval entes em pacientes com disfagia foram a hipertensão arterial e diabetes (75\%) seguido do grupo de pacientes disfagicos portadores de hipertensão arterial, diabetes e obesidade (63\%). Q uanto à chance de ter disfagia, o valor mais al to encontrado foi entre pacientes vítimas de AVC portadoras de hipertensão e diabetes com $O R$ de 3,0. Estes dados estão apresentados na T abela 1.

T abela 1 - Distribuição dos fatores de risco relacionados à presença de disfagia. Rio de Janeiro, set. 2008.

\begin{tabular}{lcccccc}
\hline \multirow{2}{*}{ Fator de risco } & \multicolumn{2}{c}{ Pacientes vítimas de AVC } & & \multicolumn{2}{c}{ M edidas } \\
\cline { 2 - 3 } & $\begin{array}{c}\text { Sem disfagia } \\
(\mathbf{n}=\mathbf{1 3})\end{array}$ & $\begin{array}{c}\text { Com disfagia } \\
(\mathbf{n}=\mathbf{1 7})\end{array}$ & & $\begin{array}{c}\text { Prevalência da } \\
\text { disfagia }(\%)\end{array}$ & $\mathbf{O R}^{*}$ \\
\hline HA & 7 & 3 & & 30 & 0,4 \\
HA e diabetes & 3 & 9 & & 75 & 3,0 \\
HA , diabetes e obesidade & 3 & 5 & & 63 & 1,7 \\
\hline
\end{tabular}

* Chance do paciente apresentar disfagia.

Legenda: AVC: acidente vascular cerebral; HA: hipertensão arterial.

$\mathrm{Na}$ T abela 2, apresentam-se os resultados da associação entre disfagia, idade e episódios de AVC. Foi feito um corte em 73 anos, considerando que a média de idade da amostra estudada foi de 73,6 anos. U sou-se o teste de $Q$ ui-quadrado $\left(\chi^{2}\right)$ com a finalidade de testar a associação entre esses atributos.

T abela 2 - Distribuição dos pacientes de acordo com a idade e episódios de AVC. Rio de Janeiro, set. 2008.

\begin{tabular}{lccc}
\hline \multirow{2}{*}{ Variáveis } & \multicolumn{2}{c}{ Pacientes vítimas de AVC } & \multirow{2}{*}{ Total } \\
\cline { 2 - 3 } & Com disfagia & Sem disfagia & \\
\hline Idade & 7 & 6 & 13 \\
A té 73 anos & 10 & 7 & 17 \\
M ais de 73 anos & 17 & 13 & 30 \\
Total & & & \\
E pisódios de AVC & 10 & 7 & 17 \\
1 & 7 & 6 & 13 \\
2 & 17 & 13 & 30 \\
Total & & & \\
\hline
\end{tabular}

Legenda: AVC: acidente vascular cerebral.

0 valor do $\chi_{\text {obs }}^{2}$ foi de 0,07 com um grau de Pearson de $0,05(p=79 \%)$, indicando que não há associação entre disfagia e idade para essa amostra estudada, ou seja, independente do aumento de idade não aumentam os casos de pacientes com disfagia pós AVC.

0 mesmo valor do $\chi_{\text {obs }}^{2}(0,07)$ foi encontrado entre os atributos disfagia e frequência de episó- dios de AVC o que permite concluir que também não foi encontrado associação neste caso.

Foi calculada a prevalência da disfagia para alimentos líquidos, sólidos e pastosos, sendo encontrados $71 \%, 44 \%$ e $38 \%$ respectivamente constatando-se que a dificuldade de deglutição para alimentos líquidos foi maior do que para alimentos sólidos ou pastosos. 


\section{DISCUSSÃO}

Os pacientes atendidos pelo SAD são aqueles que apresentam múltiplas comorbidades e precisam ser vistos por uma equipe interdisciplinar. A assistência domiciliar é o tipo de atendimento atualmente preconizado para uma população com idade superior aos 70 anos, que apresenta alta dependência de cuidados, porém não necessita de atendimento hospitalar ${ }^{(4)}$. Além disso, pela cronicidade das comorbidades, tais pacientes per manecem sob cuidados do SAD por tempo prolongado, o que reafirma a estratégia de reversão da atenção centrada em hospitais, propiciando a construção de um enfoque do cuidado domiciliar.

Foi encontrada uma prevalência de $57 \%$ de disfagia entre os pacientes com AVC, val or encontrado em outro estudo ${ }^{(8)}$. Existe uma al ta incidência de disfagia orofaríngea após um AVC isquêmico, chegando a $90 \%$ o que coincide com os dados desta amostra, uma vez que todos os pacientes disfágicos tiveram AVC isquêmico(9).

O AVC constitui o principal grupo de doenças do sistema nervoso central (SNC) em idosos (72\%) e a incidência do AVC aumenta com a idade. Dos 45 aos 54 anos essa incidência é de um para cada 1000 pacientes $^{(9)}$. Quanto ao sexo, verificouse maior frequência no gêner o feminino ${ }^{(10)}$.

Quanto à escolaridade nos pacientes do SAD, há predomínio do ensino fundamental completo (76,48\%), o que se constitui em dado relevante, considerando que uma escolaridade mais alta está relacionada ao aumento da sobrevida, ao meIhor controle de fatores de riscos para doenças cardiovasculares e a capacidade de retornar ao trabaIho(11,12).

Quanto ao status profissional, os resultados dessa pesquisa mostram que a maioria se encontra na condição de aposentado (70\%), situação provocada pela existência de al gum grau de dependência do paciente, causado pela restrição e/ ou perdas da capacidade e habilidade para desempenho de atividades profissionais, principalmente no primeiro ano após o AVC.

Verificou-se, em uma população vítima deAVC que a capacidade de voltar ao trabal ho e às atividades cotidianas ocorreu em pouco mais de $30 \%$ dos sobreviventes, causando problemas de reajustamento familiar nos âmbitos social, econômico e profissional, o que coincide com o encontrado nos pacientes do SAD ${ }^{(10,13,14) \text {. }}$

\section{Idade}

E $m$ relação à idade, o idoso tem maior risco para a disfagia pós AVC ${ }^{(15)}$. Há registros de maior incidência de disfagia em pacientes mais idosos, concluindo-se que a idade é um preditor de disfagia, havendo correlação entre disfagia e idade. A média de idade entre os pacientes disfágicos pós AVC corresponde a cerca de 74,$3 ; 73,2 ; 70,5$ anos s $^{(16-18)}$.

É consenso que o processo da deglutição é afetado como um todo pela idade, com mudanças quanto às características gerais da função. Pacientes idosos são mais propensos a apresentarem problemas iniciais de deglutição, com alta taxa de mortalidade ${ }^{(19)}$.

\section{E pisódios de AVC}

A presença de disfagia na amostra deste estudo não esteve associada à frequência de episódios de AVC $\left(\chi^{2}=0,07\right)$, tal como se obteve nos resultados. 0 comprometimento neurológico consequente ao AVC pode provocar sequelas motoras, de linguagem, fala e na dinâmica da deglutição e que mais da metade dos pacientes após um AVC apresentam disfagia.

Os sinais neurológicos variam conforme a localização do AVC no cérebro. Em geral, pacientes com AVC que envolva o hemisfério cerebral esquerdo podem apresentar dificuldades na fala e aqueles com danos ao hemisfério direito tendem a apresentar problemas de percepção e disfagia. Dessa forma, a localização do AVC é um fator mais determinante do que o número de episódios de AVC para 0 acometimento da disfagia(20).

\section{Fatores de risco}

Os fatores de risco encontrados na pesquisa for am a hipertensão ar terial, diabetes e a obesidade. A hipertensão e o diabetes aumentam o risco de desenvolvimento de um AVC em ordem de grandeza variável, e que a hipertensão arterial está presente a $60,5 \%$ dos pacientes disfágicos e em $23,3 \%$ daqueles com diabetes. 0 fator de risco prevalente encontrado em pacientes disfágicos com AVC foi a hipertensão arterial, estando presente em 63,80\% dos pacientes, o tabagismo em $60,30 \%$ e o diabetes em $13,58 \%{ }^{(16)}$.

0 diabetes de forma isolada, está associado a ocorrência de AVC isquêmico, quando comparado 
ao AVC hemorrágico. Já o D iabetes M ellitus tipo II e a hipertensão arterial estão relacionadas ao surgimento de AVC hemorrágico. 0 diabetes pode acelerar o processo de arteriosclerose, favorecendo o surgimento de AVC precocemente. Confirmando esses dados outro estudo verificou que $20 \%$ dos pacientes que sofreram AVC apresentavam diabetes $^{(16)}$.

\section{Consistência do alimento}

Os resultados junto aos pacientes atendidos pelo SAD com disfagia mostram que a dificuldade de deglutição para alimentos líquidos foi prevalente em rel ação a al imentos sólidos ou pastosos $(71 \%$, $44 \%, 38 \%$ respectivamente), caracterizando que a alteração de deglutição para alimentos líquidos é mais importante do que para os outros tipos de consistência de alimentos.

Por esse motivo, pacientes com disfagia necessitam de dietas prescritas próprias para disfágicos. A credita-se que muitos deles podem aspirar ou apresentar penetração laríngea de alimentos porque a consistência da dieta não é adequada para sua condição clínica(20).

Foi verificado que em 62.199 prescrições para identificar a dieta de pacientes disfágicos inter nados durante um ano, somente $13 \%$ eram específicas para disfagia. A dieta específica para disfagia, nessa pesquisa, era equilibrada nutricional mente com consistência pastosa e líquidos espessos, de acordo com a condição de deglutição do paciente e era fornecido aos cuidadores um manual sobre dieta e disfagia (14).

Quanto à consistência alimentar o líquido é reconhecidamente mais difícil de ser deglutido em pacientes neurologicamente acometidos ${ }^{(18,19)}$. M uitos pacientes disfágicos têm mais problemas na deglutição de líquido do que pastoso. Por essa razão, é essencial avaliar a capacidade de deglutição de alimento pastoso antes de líquido, porque esse procedimento passo a passo minimiza o risco de aspiração durante o teste e identifica pacientes que toleram a ingestão de alimentos pastosos, mas não de líquidos ${ }^{(20)}$.

A aspiração é definida como a presença de materiais gástricos ou provenientes da orofaringe no trato respiratório inferior. Diferencia-se da penetração laríngea, que é definida como a entrada de materiais da orofaringe na laringe, distalmente às pregas vocais, sem atingir traquéia ou brônquios.
E ssa difer ença é útil na medida em que, frequentemente, pode-se observar penetração sem aspiração(20).

Para uma adequada avaliação da deglutição, é necessário que o estado em que o paciente se encontra ofer eça condições seguras de avaliação, caso contrário pode-se lhe provocar um dano, sendo 0 nível de consciência um dos preditores do desenvolvimento de problemas na deglutição(16).

Estudos apontaram que no paciente disfágico, o risco para desenvolver pneumonia aspirativa é 3,17 vezes maior do que no acamado sem disfagia e 11,5 vezes maior para o risco de aspiração ${ }^{(16,20)}$.

Esses dados reforçam a necessidade de uma avaliação na fase aguda do AVC, assim como, avaliações seriadas durante o período de recuper ação, pois a disfagia e o risco de aspiração estão presentes no primeiro e no sexto mês após o AVC ${ }^{(14)}$.

Outro risco associado à disfagia é a desnutrição. E m um estudo com 50 indivíduos disfagicos, verificou-se que $49 \%$ estavam desnutridos, incidência que diminuiu para 34\% após intervenção nutricional. Então, existe uma associação significativa entre desnutrição e disfagia ${ }^{(19)}$.

A avaliação da deglutição deve aguardar a meIhora do nível de consciência e que o paciente não esteja usando ventilação mecânica, uma vez que o rebaixamento do nível de consciência elimina por si só a possibilidade de avaliação da deglutição funcional.

\section{CONCLUSÕES}

As informações obtidas acerca das características dos pacientes disfágicos encontradas talvez auxiliem o mapeamento das situações de risco referentes à disfagia durante os cuidados de enfermagem exclusivamente no ambiente domiciliar. No entanto, impõe-se a necessidade de novas investigações que demonstrem a incidência da disfagia em outras realidades além do domicilio; as complicações mais frequentes dos pacientes disfágicos vítimas de AVC, avaliem o impacto da adoção de medidas preventivas no combate á pneumonia aspirativa e desnutrição.

Algumas fragilidades da pesquisa não puderam ser superadas como a dificuldade em trabaIhar com uma população maior e os poucos estudos encontrados em bases virtuais sobre o tema, reduzindo a comparação dos dados obtidos com outras realidades. 
U m aspecto positivo foi o emprego da entrevista como técnica de coleta de dados, já que permitiu a avaliação in loco não só da condição do paciente em relação à disfagia, mas também gerou outro tipo de informação relacionada com as condições de moradia, e que serão usadas para uma próxima pesquisa, onde a partir dessas condições se desenvolvam recursos viáveis para se ensinar ao paciente e cuidador medidas que possam ser empregadas no controle da desnutrição e pneumonia.

A disfagia após o AVC afeta a vida de muitas pessoas de diversas maneiras, direta ou indiretamente, levando a implicações que incluem não só problemas de ordem biológica, mas psicológica e social também. Viver per manentemente ou por longos períodos de tempo com disfagia pode levar a alterações no processo de deglutição e a necessidade de lidar com uma linguagem corporal ou estilo de vida alterado. Por isso, é fundamental que o enfermeiro forneça, por exemplo, treinamento de deglutição para esses pacientes, indicando o tipo de alimento e em qual consistência são mais facilmente deglutidos.

A tualmente, observa-se, no mundo inteiro, a estruturação de centros de atendimento interdisciplinar ao paciente disfágico, sendo essa uma prática recente no Brasil. E sses centros reconhecem a importância da equipe multidisciplinar, o que reforça a possibilidade de 0 enfer meiro ter um papel mais ativo na soma desses esforços para o cuidado direcionado aos pacientes disfágicos.

No contexto do SAD os enfermeiros precisam se capacitar para realizarem a avaliação da disfagia nos pacientes, demonstrando conhecimento sobre suas complicações e garantirem, primariamente, a educação do paciente, do cuidador e da família, a fim de conquistar para o paciente uma alimentação segura, prevenindo a pneumonia e a desnutrição.

\section{REFERÊNCIAS}

1 A dams H P, A dams RJ, Brott T. A scientific statement from the council of the A merican Stroke Association. Stroke. 2003;34(1):1056-83.

2 Lessa I. 0 adulto brasileiro e as doenças da modernidade: epidemiologia das doenças crônicas nãotransmissíveis. São Paulo: Hucitec; 2007.

3 Farias N, Buchalla CM . A classificação inter nacional de funcionalidade, incapacidade e saúde da Organi- zação M undial da Saúde: conceitos, usos e perspectivas. Rev Bras E pidemiol. 2005;8(2):134-42.

4 Silva KL, Sena R, L eite J C, Seixas CT, G onçalves AM . Internação domiciliar no Sistema Ú nico de Saúde. Rev Saúde Pública. 2005;39(3):391-7.

5 M inistério da Saúde (BR ), Conselho N acional de Saúde, Comitê Nacional de Ética em Pesquisa em Seres Humanos. Resolução 196, de 10 de outubro de 1996: diretrizes enormas regulamentadoras de pesquisa envolvendo seres humanos. Brasília (D F); 1996.

6 Barros AF F, F abio SRC, F urkim A M . Correlação entre os achados clínicos da deglutição e os achados da tomografia computadorizada de crânio em pacientes com acidente vascular cerebral isquêmico na fase aguda da doença. Arq N europsiquiatr. 2006;64(4): 1009-14.

$7 \mathrm{M}$ artino R, Foley N, Bhogal S, Diamant N, Speechley $M, T$ easell R . D ysphagia after stroke: incidence, diagnosis and pul monary complications. Stroke[ Internet] . 2005 [ cited 2008 June 13] ;36(1):2756-2763. Available from: http:/ / stroke.ahajournals.org/ cgi/ reprint/ 36/ 12/ 2756.

8 Smithard D G, Smeeton N C, W olfe CDA. Long-term outcome after stroke: does dysphagia matter? Age A geing [ Internet] . 2007 [ cited 2008 ] une 13] ;36(1): 90-94. Available from: http:/ / ageing.oxfordjour nals. org/ cgi/ content/ full/ 36/ 1/ 90.

9 Santana BS, Fukujima M M, Oliveira R M C. Características sócio-econômicas de pacientes com acidente vascular cer ebral. A rq N europsiquiatr. 1996;54 (3):428-32.

10 Lessa I. D oenças crônicas não transmissíveis no Brasil: um desafio para a complexa tar efa da vigilância. Ciênc Saúde Colet. 2004;9(4):931-43.

$11 \mathrm{~N}$ eau, J.P. F unctional recovery and social outcome after cerebral infarction in young adults. Cerebrovasc Dis. 1998;8(5):296-302.

12 T avares DM, Pereira GA, I wamoto HH, M iranzzi SSC, Rodrigues LR, M achado ARM . Incapacidade funcional entre idosos residentes em um município do interior de $\mathrm{M}$ inas $\mathrm{G}$ erais. T exto Contexto $\mathrm{E}$ nferm. 2007;16(1):32-9.

13 PitellaJ E, D uarteJ E. Prevalência e padrão de distribuição das doenças cereberovasculares em 242 ido- 
sos, procedentes de um hospital geral, necropsiados em Belo H orizonte, $M$ inas $G$ erais, no período de 1976 a 1997. Arq Neuropsiquiatr. 2002;60(4):4755.

14 American Heart Association. Guidelines for prevention of stroke in patients with ischemic stroke or transient ischemic attack: a statement for healthcare professionals. Stroke. 2006;37(2):577-617.

15 Schelp A O, Cola PC, G atto AR, Silva RG, Carvalho $L R$. Incidência de disfagia or ofaríngea após acidente vascular encefálico em hospital público de referência. Arq N europsiquiatr. 2004;62(2):503-6.

16 M enezes FT, G onçalves M IR, Chiari BM . A daptações al imentares em adultos após um AVCI sem queixa de disfagia. Fono A tual. 2005;8(34):14-24.

\section{Endereço da autora / Dirección del autor /}

\section{Author's address:}

Lolita D opico da Silva

Rua F lordelice, 505, casa 1, Cond. Bosque dos Esquilos

22753-800, Jacarepaguá, RJ

E-mail: lolita.dopico@gmail.com
$17 \mathrm{G}$ asperin $\mathrm{D}$, Fenster seifer $L M$. As modificações do estilo de vida para hipertensos. R ev $\mathrm{G}$ aúcha E nferm. 2006;27(3):372-8.

$18 \mathrm{M}$ arik $\mathrm{P}$, Kaplan D. Aspiration pneumonia and dysphagia in the elderly. Chest. 2005;124(1):328-36.

19 H inchey J A, Shephard T, F urieK, Smith D, Wang D, Tonn S. Formal dysphagia screening protocols prevent pneumonia. Stroke [ Internet] . 2005 [ cited 2008 Aug 01] ;36(9):1972-6. Available from: http:/ / stroke.ahajournal s.org/ cgi/ content/ abstract/ 36/ 9/ 1972.

20 Correia M I, Waitzberg DL. T he impact of malnutrition on morbidity, mortality, length of hospital stay and costs evaluated through a multivariate model analysis. Clin N utr. 2003;22(3):235-40.
Recebido em: 15/ 03/ 2010

A provado em: 16/ 05/ 2010 